\title{
Operation tolerance after liver transplantation
}

\author{
Kyeong Deok Kim, Gyu-Seong Choi, Sunghae Park, Young Ju Oh, Sang Oh Yun, Manuel Lim, Eun Sung Jeong, Ji Eun Kwon, \\ Jaehun Yang, Jinsoo Rhu, Jong Man Kim, Jae-Won Joh
}

Department of Surgery-Transplantation, Samsung Medical Center, Seoul, Korea

Background: Advances in immunosuppressive therapy have improved clinical outcomes after liver transplantation (LT) over the years. On the other hand, there are serious risks associated with immune suppression such as opportunistic infections, de novo malignancies, cardiovascular disease, metabolic disorders, kidneys, and other complications. Minimization or withdrawal of immunosuppression may overcome these problems. This study aimed to identify the patients who were successful for immunosuppression minimization or withdrawal after LT.

Methods: All patients who underwent LT from May 1996 and December 2016 were retrospectively reviewed. The patients with immunosuppression withdrawal or minimization for monotherapy or dual therapy with minimum dose were included. And the patients with rejection episodes within the last 5 years and under 18 years of age at the time of LT were excluded.

Results: The 57 recipients were included by immunosuppression minimization, of which four (7.0\%) were immunosuppression withdrawal. Living donor LT was 35 patients $(61.4 \%)$. There were 41 patients $(71.9 \%)$ immunosuppression minimizing with monotherapy and 16 patients $(28.1 \%)$ immunosuppression minimizing with dual therapy. Immunosuppression minimization with a calcineurin inhibitor and mycophenolate mofetil was performed in $30(52.6 \%)$ and 37 patients $(64.9 \%)$, respectively, mostly monotherapy with these or dual therapy with this combination. The average graft-recipient weight ratio was $1.43,1.13$ for living donor LT and 2.31 for deceased donor LT. The median time to minimize immunosuppression was 5.2 years and the median follow-up period was 14.2 years.

Conclusions: Early immunosuppression minimization is feasible in selected liver recipients, while complete withdrawal is successful in only a small proportion.

Corresponding author: Gyu-Seong Choi

E-mail: gyuseong.choi@samsung.com

(c) The Korean Society for Transplantation

This is an Open Access article distributed under the terms of the Creative Commons Attribution Non-Commercial License (http://creativecommons.org/licenses/by-nc/4.0/) which permits unrestricted non-commercial use, distribution, and reproduction in any medium, provided the original work is properly cited. 